\title{
FACTORES DE RIESGO ASOCIADOS A MORTALIDAD MATERNA EN LA REGIÓN DE ICA, PERÚ 2001-2005.
}

\author{
Jorge Ybaseta-Medina ${ }^{\text {1,2,a,b. }}$ \\ 1. Facultad de Medicina. Universidad Nacional San Luis Gonzaga. Ica. Perú. \\ 2. Hospital Santa María del Socorro. Ica, Perú. \\ a. Médico Ginecólogo, b. Doctor en Medicina
}

\section{RESUMEN}

Objetivo: Determinar los factores de riesgo asociados a mortalidad materna en la región de Ica, Perú durante los años 20012005. Material y Métodos: Se realizó un estudio de tipo caso y control, el grupo de casos fueron 20 mujeres fallecidas, comparado con un segundo grupo control de 40 mujeres que sobrevivieron a complicaciones en el embarazo, parto y puerperio. En el análisis para cada variable de interés tuvo como base la estimación de Razón de Disparidad OR y sus intervalos de confianza en 95\%. Resultados: Los factores asociados a mortalidad materna fueron parto atendido por partera OR 2,27; IC (1,20 - 93,56); no saber reconocer los signos de alarma OR 7,21; IC $(1,83$ - 161,53) el embarazo no deseado OR 6,93; IC (2.01 - 23,85); presentar complicaciones prenatales OR 2,15; IC $(1,52-8,80)$; pobreza extrema OR 2,73; IC $(1,68-11,00)$, contar con servicios públicos inadecuados OR 3,50 ; IC $(1,11-11,02)$, vivir con una pareja OR 4,64; IC $(1,17$ 18,36); controles prenatales por no profesional OR 2,05; IC $(1,12-34,63)$ y pacientes referidos en estado crítico OR 2,05 IC $(1,68-6,22)$. Conclusiones: Existe una serie de factores de riesgo de mortalidad materna que pueden ser modificables lo que demanda una mayor intervención de profesionales y autoridades de la salud en esta región. (Rev. méd. panacea 2011; $1: 9-13)$.

Palabras clave: Mortalidad Materna, Factores de Riesgo, complicaciones del embarazo, Perú (fuente: DeCS BIREME).

\section{RISK FACTORS ASSOCIATED WITH MATERNAL MORTALITY IN ICA REGION , PERU 2001-2005.}

\begin{abstract}
Objective: Determine the risk factors associated with maternal mortality in Ica region, Peru during the years 2001-2005. Material and Methods: It was realized a case-control study, the groups of cases were 20 women died, compared with a second control group of 40 women that survived to the complications in pregnancy, childbirth and postpartum. In the analysis for each variable of interest and this had as basis the estimation of Reason of Disparity OR and confidence intervals at $95 \%$. Results: Factors associated with maternal mortality were births were attended by midwives OR 2.27, Cl (1.20 - 93.56), not know to recognize the signs of alarm OR 7.21, Cl (1.83 - 161.53) unwanted pregnancy OR 6.93, $\mathrm{Cl}(2.01-23.85)$, have prenatal complications OR 2.15, $\mathrm{Cl}$ (1.52- 8.80), an extreme poverty OR 2.73, $\mathrm{Cl}(1.68$ - 11.00), have inadequate public services OR 3.50, $\mathrm{Cl}(1.11$ - 11.02), living with a partner OR 4.64, $\mathrm{Cl}(1.17$ - 18.36) prenatal controls without respective professionals OR 2.05, Cl (1.12 - 34.63) and referred patients in critical conditions OR $2.05 \mathrm{Cl}(1.68$ - 6.22$)$. Conclusions: There are a series of risk factors about maternal mortality that can be modified which require a high intervention of professionals and health authorities in this region. (Rev. méd. panacea 2011; 1:9-13).
\end{abstract}

Key words: Maternal Mortality, Risk Factors, pregnancy complications, Peru (source: MeSH NLM).

\section{INTRODUCCIÓN}

La Organización Mundial de la Salud (OMS) establece que se producen 585.000 muertes maternas por año en el mundo entero, de las cuales el $99 \%$ ocurren en países subdesarrollados, principalmente en Asia, África y América Latina $^{(1)}$.
En las Américas sólo dos países tienen tasas de mortalidad materna inferior a 10 por 100.000 nacidos vivos, estos son Estados Unidos y Canadá, consideradas como las tasas más bajas del mundo debido a la cobertura universal y acceso igualitario de toda la población a los servicios de salud. Países como Cuba, Costa Rica, Chile, Uruguay y México tienen tasas de mortalidad materna intermedias que se ubican entre 20-49 por 100.000 nacidos 
vivos; el resto de los países de América como Bolivia, Guatemala, Haití, Honduras y Perú registraron los valores más altos, más de 150 muertes maternas por 100000 nacidos vivos ${ }^{(2)}$.

Los efectos de la pobreza en las muertes maternas se ven potencializados por factores socioculturales que generalmente, no son previstos ni tomados en cuenta al diseñar e instrumentar programas de salud ${ }^{(3)}$.

La OMS establece como mortalidad materna a toda muerte de una mujer mientras está gestando o dentro de los 42 días siguientes a la terminación del mismo, debido a cualquier causa relacionada o agravada con el embarazo o su atención, pero no por causas accidentales o incidentales $^{(4)}$.

La muerte materna se clasifica en obstétrica directa e indirecta. Las directas; son las que resultan de complicaciones obstétricas de la gestación, parto o puerperio, de intervenciones, omisiones, tratamiento incorrecto o de la cadena de acontecimientos originada en cualquiera de las circunstancias mencionadas. Las muertes maternas obstétricas indirectas: son las que resultan de una enfermedad existente desde antes del embarazo o de una enfermedad que evoluciona durante el mismo, no debidas a causas obstétricas directas pero si agravadas por los efectos fisiológicos del embarazo ${ }^{(5,6)}$.

En el Perú de acuerdo a estimaciones realizadas el 2002 se habrían producido 1027 muertes maternas lo que significa una razón de mortalidad de 163 por cada 100,000 nacidos vivos. La mala situación de la salud materna y el bajo acceso de las gestantes a los servicios de salud son las principales causas de estas muertes, siendo su incidencia mayor en zonas deprimidas, dónde la mitad de las mujeres en edad reproductiva se encuentran en situación de pobreza, el 19\% no sabe dónde acudir cuando tienen problemas de salud, el 30\% residen en áreas rurales alejadas $^{(7)}$.

La región de Ica se encuentra ubicada en el mapa de pobreza de nuestro país en el estrato regularmente pobre y no es ajeno a las características sociales, culturales y económicas de la que presenta el país; entre los años 2001 al 2005 ocurrieron 25 muertes maternas todas probablemente evitables debido a la existencia de tecnología disponible para diagnosticar y prevenir, además de no faltar medios para referir oportunamente a la madre a niveles de atención más complejos ${ }^{(8)}$.

El objetivo de esta investigación fue determinar los factores asociados a la mortalidad materna en la Región de Ica, Perú durante los años 2001 y 2005.

\section{MATERIAL Y MÉTODOS}

Se realizó un estudio observacional, transversal y analítico de tipo casos y controles.

Mediante un muestreo no probabilística se incluyeron como Casos a 20 del total de 25 muertes maternas registradas por el Sistema de Vigilancia Epidemiológica de la Dirección Regional de Salud de lca desde el año 2001 al 2005, se revisaron las historias clínicas correspondientes siendo excluidos cinco casos por no disponer de información completa.

El tamaño muestral para los Controles fueron 40 mujeres que sobrevivieron a complicaciones graves del embarazo, parto y puerperio, los mismos que debían estar encuadrados en intervalos gestacionales antes de las 22 semanas, entre las 23 y 36 semanas y mayor a las 36 semanas de gestación, y que también cumplan con similares necesidades básicas insatisfechas, residencia en el mismo distrito y provincia, presentando la complicación el mismo año de la muerte materna.

Se aplicó además un cuestionario semiestructurado mediante entrevista directa a la pareja, familiares, personas relacionadas $y / 0$ personal de salud que la atendieron de los casos de muerte materna y en los controles a la mujer por el mismo investigador. Este instrumento recogió la información acerca de los factores asociados al evento de la muerte materna así como de sus complicaciones.

Se establecieron mecanismos de control de calidad y confiabilidad de la información, mediante el cruce de respuestas entre informantes, haciendo el juicio discriminatorio mediante un análisis cruzado, corroborado con la historia clínica. Posteriormente se recurrió a dos expertos del Ministerio de Salud, uno para los casos y el otro para los controles quienes revisaron la información recogida.

El análisis estadístico de los datos fue analizado mediante el software SPSS versión 14 y EPIINFO 6.04. Se aplicó para cada variable estadística descriptiva y para el análisis de los factores asociados los Odds Ratio con sus respectivos intervalos de confianza al 95\%.

\section{RESULTADOS}

Las tasas de mortalidad materna por 100,000 nacidos vivos en los años 2001 a 2005 fueron de 7,37; 27,47; 51,$77 ; 45,50$ y 53,76 respectivamente. 
El promedio de edad para los casos fue de 30,4 años y para los controles de 28,1 años. El $60 \%$ de las madres eran convivientes y $41,7 \%$ procedían de Ica. Las principales causas de mortalidad materna que se encontraron fueron: toxemia y abortos ambas con $41,2 \%$ (Tabla 1.)

Tabla 1. Características de la población estudiada

\begin{tabular}{|c|c|c|}
\hline Variable & $\mathbf{N}$ & (\%) \\
\hline \multicolumn{3}{|l|}{ Edad } \\
\hline $15-19$ & 9 & $(15,0)$ \\
\hline $20-24$ & 9 & $(15,0)$ \\
\hline $25-29$ & 14 & $(23,3)$ \\
\hline $30-34$ & 12 & $(20,0)$ \\
\hline $35-39$ & 10 & $(16,7)$ \\
\hline $40-44$ & 6 & $(10,0)$ \\
\hline \multicolumn{3}{|l|}{ Estado civil } \\
\hline Conviviente & 36 & $(60,0)$ \\
\hline Casada & 12 & $(20,0)$ \\
\hline Soltera & 8 & $(13,3)$ \\
\hline Separada & 4 & $(6,7)$ \\
\hline \multicolumn{3}{|l|}{ Procedencia } \\
\hline Ica & 25 & $(41,7)$ \\
\hline Pisco & 22 & $(36,7)$ \\
\hline Chincha & 10 & $(16,7)$ \\
\hline Nazca & 3 & $(5,0)$ \\
\hline \multicolumn{3}{|c|}{ Causa de mortalidad materna } \\
\hline \multicolumn{3}{|l|}{ Directa } \\
\hline Toxemia & 21 & $(41,2)$ \\
\hline Aborto & 21 & $(41,2)$ \\
\hline Infección & 6 & $(11,8)$ \\
\hline Hemorragia & 3 & $(5,9)$ \\
\hline \multicolumn{3}{|l|}{ Indirecta } \\
\hline Pielonefritis & 6 & $(66,7)$ \\
\hline Tuberculosis & 3 & $(33,3)$ \\
\hline
\end{tabular}

Se encontraron como factores asociados a mortalidad materna el parto atendido por partera OR 2,27; IC (1,20 93,56); dentro de las características de la madre, no saber reconocer los signos de alarma OR 7,21 ; IC $(1,83$ 161,53) el embarazo no deseado OR 6,93; IC (2.01 $23,85)$ y presentar complicaciones prenatales OR 2,15 ; IC $(1,52-8,80)$; según los factores socioculturales estuvieron relacionados la pobreza extrema OR 2,73; IC (1,68 $11,00)$, contar con servicios públicos inadecuados OR 3,50 ; IC $(1,11$ - 11,02), vivir con una pareja OR 4,64; IC ( 1,17 18,36); los factores de servicios de salud relacionados fueron: controles prenatales atendidos por no profesional
OR 2,05 ; IC $(1,12-34,63)$ y pacientes referidos en estado crítico OR 2,05 IC (1,68-6,22) (Tabla 2).

\section{DISCUSIÓN}

La mayor proporción de mortalidad materna entre los 21 y 34 años fue consistente con el hallado por la DGE del MINSA entre los años 2001 a 2003, sin embargo los grupos de mayor riesgo de morbimortalidad materna están entre las edades menores a 19 años y mayores de $35^{(5,12)}$.

Según la distribución de los casos de mortalidad materna registradas en hospitales de la región, en su mayoría tienen como procedencia Ica y Pisco, sin embargo debe considerarse que la provincia de Pisco presenta menor población que la provincia de Ica, según el Instituto Nacional de Estadística e Informática en el año 2000 la provincia de Pisco tenía una población de 121,097 mientras que la provincia de Ica 290,965 habitantes por lo que la tasa de mortalidad materna seria más alta en la provincia de Pisco, debemos considerar además que muchos de los casos registrados en el Hospital Regional de Ica fueron derivados de provincias de los departamento de Huancavelica y Ayacucho ${ }^{(9,10)}$.

Con respecto a la relación en la atención del parto entre las parteras y la mortalidad materna, Benavides en su estudio a nivel nacional hace mención que las parteras tradicionales aportan de dos maneras a la reducción de la mortalidad materna, primero sí recibe capacitación de parto limpio y segundo si como aliada estratégica de los servicios de salud cumple una función de bisagra intercultural ${ }^{(11)}$.

Con respecto al embarazo no deseado, en el presente estudio existe clara asociación con la mortalidad materna difiere de la investigación de Torres y colaboradores en Medellín quienes no encuentran para el embarazo no deseado una relación significativa ${ }^{(13)}$.

En el presente estudio las complicaciones prenatales tienen una asociación significativa con la mortalidad materna resultados que son consistentes con múltiples estudios realizados en diversas latitudes de Latinoamérica, por lo que es necesario fortalecer la prestación de los servicios de control prenatal y atención del parto por personal profesional calificado, con el fin de reducir las complicaciones durante el embarazo, el parto y el puerperio. Mejorando además la calidad de los servicios de planificación familiar con el fin de reducir las posibilidades de embarazos no deseados ${ }^{(12,13)}$. 
Tabla 2. Factores asociados a mortalidad materna en la Región de de Ica 2001-2005.

\begin{tabular}{|c|c|c|}
\hline Variables & OR & IC (95\%) \\
\hline \multicolumn{3}{|l|}{ Características del parto } \\
\hline Parto domiciliario & 2,27 & $0,0-93,56$ \\
\hline Parto Distócico* & 1,84 & $0,26-15,78$ \\
\hline Parto atendido por partera & 2,27 & $1,20-93,56$ \\
\hline \multicolumn{3}{|l|}{ Características de la madre } \\
\hline No reconoció los signos de alarma & 7,21 & $1,83-161,53$ \\
\hline Espacio intergenésico menor de 2 años & 0,43 & $0,14-1,30$ \\
\hline Edad menor de 20 y mayor de 35 & 0,66 & $0,22-2,00$ \\
\hline Paridad más de 5 & 0,37 & $0,04-3,39$ \\
\hline Embarazo no deseado & 6,93 & $2,01-23,85$ \\
\hline Complicaciones prenatales & 2,15 & $1,52-8,80$ \\
\hline Antecedente de aborto & 1,22 & $0,42-3,58$ \\
\hline Antecedentes patológicos & 2,27 & $0,76-6,78$ \\
\hline \multicolumn{3}{|l|}{ Características socioculturales } \\
\hline Ama de casa & 0,33 & $0,11-1,03$ \\
\hline Grado de Instrucción primaria & 1,00 & $0,31-3,23$ \\
\hline Pobreza extrema & 2,73 & $1,68-11,00$ \\
\hline Vivienda inadecuadas & 1,00 & $0,32-3,08$ \\
\hline Servicios públicos inadecuados & 3,50 & $1,11-11,02$ \\
\hline Hacinamiento & 0,49 & $0,16-1,46$ \\
\hline Hijos con inasistencia escolar & 0,42 & $0,12-1,48$ \\
\hline Dependencia económica & 1,11 & $0,38-3,26$ \\
\hline Más de 5 integrantes familiares & 0,76 & $0,17-3,01$ \\
\hline Vive con su pareja & 4,64 & $1,17-18,36$ \\
\hline \multicolumn{3}{|l|}{ Características del servicio de salud } \\
\hline Sin control prenatal & 0,82 & $0,27 \quad 2,42$ \\
\hline Control prenatal por no profesional & 2,05 & $1,1234,63$ \\
\hline Otro lugar de control prenatal & 0,82 & 0,27 \\
\hline Paciente referido en estado crítico & 2,05 & $1,68 \quad 6,22$ \\
\hline
\end{tabular}

* (Cesárea + vaginal con complicaciones).

Estos resultados indican una asociación entre mortalidad materna y disponibilidad inadecuada de servicios públicos, no guardando relación con otras necesidades básicas insatisfechas con que se valoró la pobreza extrema, como vivienda inadecuada, hacinamiento, inasistencia escolar, dependencia económica y número de integrantes familiares mayor de cinco. Sin embargo el estudio de Torres y colaboradores en Medellín Colombia hallan que el hacinamiento tiene un riesgo significativo de este indicador socioeconómico con la mortalidad materna ${ }^{(13)}$.
Vivir con la pareja es un predictor de la mortalidad materna concordante con la estadística manejada por la Dirección General de Epidemiología del Ministerio de Salud que informa que entre los años 2000 y 2002 el $81,8 \%$ de muertes maternas tenían una relación estable de pareja, mientras un $13,9 \%$ es registrada en mujeres sin pareja, estos resultados tienen relación sobre todo con mujeres pobres, no protegidas contra el embarazo no deseado y expuestas a una alta tasa de fecundidad ${ }^{(7)}$.

La mortalidad materna asociada a la atención del parto por otra persona no capacitada guardo relación con la investigación de Urrego y Col. realizada en la localidad de Kennedy- Bogotà, refiriendo que la atención del parto requiere del personal de salud debidamente entrenado de manera que se pueda identificar y controlar los riesgos de forma oportuna, así mismo señala que la experticia del profesional está altamente asociada con el nivel de atención especializada del establecimiento de salud ${ }^{(12)}$.

Pacientes referidas en estado crítico está asociado con mortalidad materna concordante con el estudio de Benavides quien atribuye las causas de muerte debido a que las gestantes hacen uso de los servicios de salud de baja complejidad cuando es demasiado tarde y porque los servicios de salud no están preparados para resolver emergencias totalmente imprevistas lo que sugiere la necesidad de fortalecer la promoción y reconocimiento de los signos de peligro de mortalidad materna a través de los trabajadores de salud de la región establecer la referencia oportuna de las complicaciones maternas evitando que los pacientes sean trasladados en estado crítico al Hospital regional de Ica ${ }^{(14,16)}$.

Se recomienda promover la aplicación de una atención íntegra en salud, con equidad y organización que sirva como sustento para el desarrollo de estrategias y actividades que puedan definirse a nivel regional, en función de contribuir a la reducción de este problema de salud pública. Asimismo fortalecer comités de análisis de mortalidad materna de cada institución que analice los casos de muerte materna y lanzar un plan de mejoras para evitar en el futuro muertes similares.

Recibido: 11-4-11 Aprobado: 24-4-11

Financiamiento: El estudio fue autofinanciado

Conflictos de interés: El autor declara no tener conflictos de interés en la publicación de este artículo. 


\section{REFERENCIAS BIBLIOGRÁFICAS}

1. Brito Hurtado, Julio G, Cabrera Carlos, Gutiérrez Orlando et al. Mortalidad materna en la maternidad "Concepción Palacios": 1982-1991. Rev Obstet Ginecol Venezuela 2007; 67(1):31-39.

2. Elu MDC. Mortalidad Materna: Una tragedia evitable. Perinatol Reprod Hum 2004;18(1):44-52.

3. Torres Y, Montoya L. Cuartas J, Osorio G. Factores asociados a mortalidad materna- estudio de casos y controles. Medellín 2001-2003. Rev CES Med. 2005; 19(2):19-45

4. Ministerio de Salud. Mortalidad materna en el Perú. Lima Perú 2003.

5. Perez S, Donoso S. Obstetrícia. 2002. Cuarta Edición. Mediterráneo.

6. Mongrut-Steane A. Tratado de Obstetricia. Cuarta Edición. Lima- Perú. 2000.

7. Ministerio de Salud. Análisis de la Situación de Salud del Perú 2003. Lima, Perú. 2004.

8. Ministerio de Salud. Análisis de la Situación de Salud del departamento de Ica. 2004. Oficina de Epidemiología.

9. Dirección Regional de Salud. Evaluación del Plan Estratégico de Intervención para la Reducción de la Mortalidad Materna en la Libertad 2001 - 2003.

10. Arcos J. Mortalidad Materna en el Hospital Regional Base de Ica 1967-1986 [Tesis para optar el grado de Magíster en Medicina]. Lima: Universidad Cayetano Heredia; 1988.
11. Benavides B. Reducción de la mortalidad materna en el Perú ¿Por dónde empezar?. An Fac Med 2001; 63(1): 215-217.

12. Urrego Z, Gonzáles E, Fernández R. Evaluación del Control Prenatal en el Hospital Kennedy Primer Nivel E.S.E. Bogotá 1999-2000; Revista de la Federación Panamericana de Asociaciones de Facultades (Escuelas) de Medicina 2001. 1(4):p12. http://www.fepafem.org.ve/investigaciones/pdf/control \%20prenat.pdf Consultado Enero 10, 2004.

13. Torres Y, Montoya L. Cuartas J, Osorio G. Factores asociados a mortalidad materna- estudio de casos y controles. Medellín 2001-2003. Rev CES Med 2005; 19(2) : 19-45.

14. Organización Panamericana de la Salud. Promoviendo la Salud en las Américas. Boletín Epidemiológico. Vol. 25 № 2. Junio del 2004.

15. Herrera M. Mortalidad materna en el mundo. Rev Chil Obstet Ginecol 2003; 68(6):536-543.

16. Ministerio de Salud. Lineamientos de política sectorial para el periodo 2002 - 2012. principios fundamentales para el plan estratégico sectorial del quinquenio agosto 2001, julio 2006. Lima, Perú.

\section{Correspondencia:}

Dr. Jorge Ybaseta Medina

Correo electrónico: jorgeybaseta@yahoo.es 